\title{
Neuroblastoma in adults
}

\author{
P. H. Rowe \\ M.A., M.B., B.Chir.
}

\author{
J. J. ORAM \\ M.R.C.P., D.Obst., R.C.O.G.
}

\author{
G. W. SCOTT \\ M.D., F.R.C.P. \\ Guy's Hospital, London, S.E.1
}

\begin{abstract}
Summary
Two cases of neuroblastoma are described in patients who were both more than 25 years old when their symptoms first appeared. The behaviour of this tumour appears less aggressive in adults than in children, but the cases presented illustrate its resistance to chemotherapy and radiotherapy, and surgery is suggested as the first line of treatment in adults, even if the growth cannot be removed completely.
\end{abstract}

\section{Introduction}

Neuroblastoma is a relatively common form of malignant tumour in children but it is extremely rare in adults.

Mackay, Luna and Butler (1976) presented the electronmicroscope observations in 9 patients whose ages ranged from 18 to 72 years.

Two cases of neuroblastoma first presenting in patients over the age of 25 years are now described.

\section{Case 1}

A 29-year-old woman presented with a 3-month history of swelling in the right flank. At laparotomy an inoperable tumour of the adrenal gland was discovered, later shown to be a neuroblastoma of moderate differentiation. Radiotherapy produced slight regression in tumour size. She presented again 3 years later with a mass in the right hypochondrium. A liver scan showed metastases in the right lobe of the liver. Cytotoxic therapy of vincristine, cyclophosphamide and adriamycin was commenced but with no success, and a malignant pleural effusion formed. Despite further courses of radiotherapy and cytotoxic therapy she died 4 years and 9 months after her first admission. No post-mortem was performed.

\section{Case 2}

A 26-year-old woman presented at another hospital with colicky pains in the right loin. Intravenous pyelography (IVP) demonstrated a soft tissue swelling over the psoas muscle, tilting the axis of the right kidney upwards. A subsequent IVP
3 months later demonstrated a further mass in the paravertebral region at the level of T11, no calcification was seen. These masses were interpreted as ganglioneuromas, however, no therapy was instituted as the patient did not attend for follow-up appointments.

She presented to the authors 2 years and 5 months after the first presentation with a mass on the left side of the neck and loss of weight. On examination there were 2 firm encapsulated masses in the left anterior triangle of the neck. There was also a fullness in the right iliac fossa, rectal and vaginal examination was normal. Routine blood investigations were all normal, apart from an elevated ESR of $96 \mathrm{~mm}$ in the first hour. An IVP showed enlargement of the soft tissue swellings originally seen at the other hospital; they extended to above the diaphragm into the posterior mediastinum, and this finding was confirmed by the chest radiograph. A gallium scan showed no abnormal uptake in the tumour, and a liver and spleen scan was normal. The 24-hr urinary catecholamine levels were markedly elevated: methoxy-hydroxymandelic acid was $22.7 \mathrm{mmol} / \mathrm{mol}$ creatinine (normal 2.4 ). The mass in the neck was biopsied and found to be a well differentiated neuroblastoma.

Cytotoxic therapy of adriamycin, vincristine and cyclophosphamide was started; however no regression in tumour size was seen after one month. She then presented with continuous pain in the right iliac fossa, on examination a mass was palpable and there were signs of compression of the right iliohypogastric and ilio-inguinal nerves by the tumour. Cytotoxic therapy was discontinued and radiotherapy (4000 rad) given to the neck and right iliac fossa. This too produced no change in the tumour size or in the urinary catecholamine levels. There was no response either to further weekly courses of cytotoxic drugs.

A scan of the lower thorax and abdomen using computerized axial tomography showed the tumour to be extending along the right posterior wall, paravertebrally, from the lower chest at the level 
of T11-12 to the pelvic brim. It completely obscured and enveloped the inferior vena cava, aorta and other para-aortic tissues. There were no liver or bony metastases identified.

On the basis of this information and its total resistance to other forms of therapy, it was decided to perform a laparotomy to resect as much tumour as possible. Seven months after presenting to the authors, this operation was performed through a thoraco-abdominal incision and $90 \%$ of the tumour was excised. The findings at operation confirmed the results of the scan. There were no postoperative complications. Three months later the masses in her neck were excised. All the specimens resected were found to be differentiated neuroblastoma. At present (1978) she is well and there is no evidence of further metastases.

\section{Discussion}

The cases presented are of 2 adults with adrenal neuroblastoma, one survived 4 years and 8 months, and the other is well after 3 years. It would appear that the tumour is less aggressive in adults than in children, where the disease-free survival rate remains at less than $30 \%$ even in major paediatric oncology centres. The adrenal neuroblastoma in children has a far worse prognosis than do tumours originating outside the adrenal gland (Kinnier Wilson and Draper, 1974; Hope-Stone, 1961). Mackay et al. (1976) have also noted that the site of the tumour in adults may differ from locations in which it commonly occurs in children.

The possible therapy for neuroblastoma in any age group includes surgery, radiotherapy (Philips, 1953), chemotherapy (Tan et al., 1973; Sullivan et al., 1969; James et al., 1965), and vitamin $B_{12}$ (Bodian, 1959). The latter has now been disproved (Kinnier Wilson and Draper, 1974; Sawitsky and Deposito, 1965; D’Angio, Evans and Koop, 1971). The 3-year prognosis for children with adrenal neuroblastoma using radiotherapy and chemotherapy (nitrogen mustard) was 12.4-17\% (Bodian, 1959; Philips, 1953); and using more modern chemotherapy (vincristine and cyclophosphamide) was $23 \%$ (Kinnier Wilson and Draper, 1974). There have been no reported trials of any therapy in adults. Both these cases showed little or no response to radiotherapy and chemotherapy, and this, together with the less aggressive nature of the tumour, leads the authors to support the view of Wittenborg (1950) and Mackay et al. (1976) that surgery should be considered as the first method of treatment, even if the growth cannot be removed completely.

\section{References}

Bodian, M. (1959) Neuroblastoma. Pediatric Clinics of North America, 6, 449.

D'Angio, G.J., Evans, A.E. \& Koop, C.E. (1971) Special pattern of widespread neuroblastoma with a favourable prognosis. Lancet, i, 1046.

Hope-Stone, H.F. (1961) Extra-adrenal neuroblastoma. British Journal of Surgery, 48, 424.

James, D.H., Hustu, O., WrenN, E.L. \& Pinkel, D. (1965) Combination chemotherapy of childhood neuroblastoma. Journal of the American Medical Association, 194, 103.

KinNier Wilson, L.M. \& DRAPER, G.J. (1974) Neuroblastoma, its natural history and prognosis. Study of 487 cases. British Medical Journal, 3, 301.

Mackay, B., Luna, M.A. \& Butler, J.J. (1976) Adult neuroblastoma: electronmicroscopic observations in nine cases. Cancer. New York, Philadelphia, etc., 37, 1334.

Philips, R. (1953) Neuroblastoma. Annals of the Royal College of Surgeons of England, 12, 29.

SAwitsky, A. \& Deposito, F. (1965) A survey of American experience with vitamin $B_{12}$ therapy of neuroblastomace Journal of Pediatrics, 67, 99.

Sullivan, M.P., Nora, A.H., Kulapongs, P., Lane, D.M., Windmiller, J. \& Thurman, W.G. (1969) Evaluation of vincristine sulphate and cyclophosphamide chemotherapy for metastatic neuroblastoma. Pediatrics, 44, 685.

Tan, C., Etcubanas, E., Wollner, N., Rosen, G., Gilladoga, A., Shovel, J., Lois Murphy, M. \& Krakoff, I.H. (1973) Adriamycin. An antitumor antibiotic in the treatment of neoplastic disease. Cancer. New York, Philadelphia, etc., 32, 9.

WitTENBORG, M.H. (1950) Roentgen therapy in neuroblastoma. Review of 75 cases. Radiology, 54, 679 . 